\title{
Statistical process control analysis based on software Q-das
}

\author{
Wu Guoqing*, Luo Yiping, Ren Hongjuan \\ Faculty of Automotive Engineering, Shanghai University of Engineering Science, Shanghai, China \\ Email address: \\ 519830568@qq.com (Wu Guoqing), lyp777@sina.com (Luo Yiping)
}

To cite this article:

Wu Guoqing, Luo Yiping, Ren Hongjuan. Statistical Process Control Analysis Based on Software Q-Das. American Journal of Theoretical and Applied Statistics. Vol. 3, No. 4, 2014, pp. 90-95. doi: 10.11648/j.ajtas.20140304.12

\begin{abstract}
The software Q-das used in statistical analysis of the production process can realize the real-time monitoring during the production process. So as to effectively support the needs of the factory in data management, long-term backup and automatic report generation. This paper tells some specific evaluation method of statistical quality control, such as single value chart, control charts, process capability index, etc. Through these configurations of figures, tables, and statistical analysis, "correctly observe" the entire production process. Control and suppress the fluctuations that occur during production. At last, this paper tells the Q-das software in statistical process control analysis application based on a specific example.
\end{abstract}

Keywords: Statistical Process Control, Q-Das Software, Evaluation Method

\section{Introduction}

In the production process, the fundamentals such as people, machines, materials, methods, and environment, etc lead to fluctuations in the machining accuracy, Wherein the normal fluctuations have less impact on product quality, technically inevitable, while abnormal fluctuations has more impact on product quality, evitable through process control.

Statistical process control (referred to SPC) is a process control technology using mathematical statistical methods. Since the U.S. Bell Labs, Dr. Hugh Hart proposed control charts at 1924; Statistical process control has been widely applied to quality management. After 90 years of development, process control continues to produce many kinds of new methods in practice. The quality control of enterprise has not limited to sampling inspection, but pursue sustainable stability of the entire production process through value chart individuals, histogram, control charts, process capability index, etc.

\section{Statistical Process Control Methods and Basic Principles}

The method of statistical process control has value chart individuals, histogram, control charts, process capability index, etc.

\subsection{Value Chart Individuals}

A value chart individual is also known as running chart. $\mathrm{X}$ axis represents time or the number of value. $Y$ axis represents measurements. The whole chart gives the manufacturing process information changed with the $\mathrm{X}$ axis.

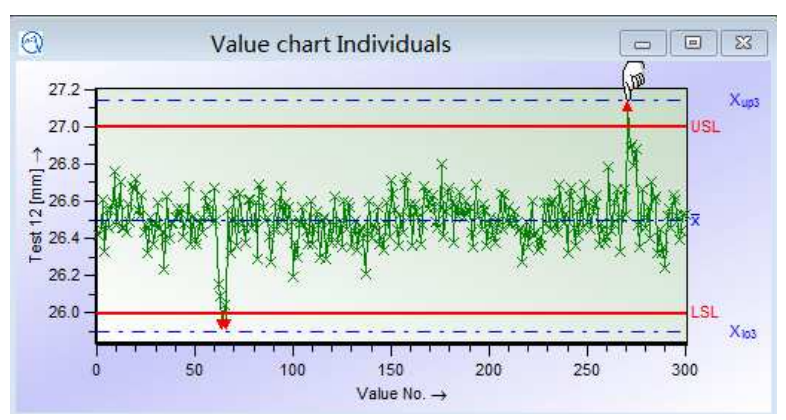

Fig 2.1. Value chart individuals

From the figure1.1 we can see there are three obvious unusual points. In the Q-das software, we can check the abnormal data information by clicking this point through the hand-shaped icon. Thereby discriminate this abnormal point is due to the systematic error or random error. And then make a judgment that whether eliminates the abnormal 
point or not. This figure can also be helpful to identify which is the main reason that affects the process performance. This can help us to find the main reason as quickly as possible and eliminate them so that the ability of the process will be improved.

\subsection{Histogram Individuals}

\subsubsection{Brief Introduction}

The frequency is the vertical axis and the observed data is the horizontal axis in Histogram. Class interval is the bottom edges and the frequency of observed data falling into each group is height. Draw a series of rectangles based on these. It provides a lot of convenience to the observation of the measured value. Height of the rectangle can help people to visually see the distribution pattern of the quality characteristics. From the histogram we can see the distribution of the data falling in each group. Find out problem areas in a narrow range based on these abnormal changes.

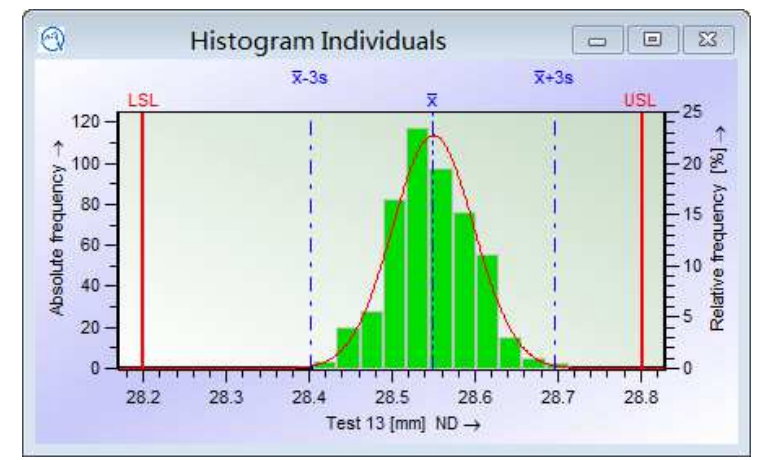

Fig 2.2. Histogram individuals

Q-DAS database will automatically match the best distribution model based on the user's assessment settings or Q-DAS recommended statistical analysis mode. From Fig2.2 we can see the gap of the data distribution between both sides of the center line is large. The distribution of the data on the left is scattered relative to the data on the right. The fluctuations of the data on the left are lager. We can take appropriate and effective measures to adjust the data of both sides in order to make it not only closer to the center line but also smooth fluctuations.

\subsubsection{Histogram Classification}

(1)Normal type (Fig 2.3(a)): process is in a steady state and run normally.

(2)Deviation type (Fig 2.3(b)): some characteristics value distribution with the requirement of geometric tolerance often show deviation type. Because of the restricted lower tolerance limit (such as unilateral geometric tolerance) or certain processing habits (such as small hole machining), processing size distribution is easy to left. When the upper tolerance limit is restricted or machines the cylindrical of axis, processing size distribution is easy to right form.

(3)Bimodal type (Fig2.3(c)): the mainly reason is that the data comes from a different overall and the two distributions of data mix together. At this time the data should be stratified.

(4)Lonely island type (Fig 2.3(d)): the main reason is the abnormal situation in the production process, such as error of measurement tools, tool wear severely; there are unskilled workers for a short post, careless operation, mixed with different specifications, etc.

(5)Flat type (Fig2.2 (e)): there are many slow factors in the production process, such as:tools wear slowly, the fatigue operator, etc.

(6)Sawtooth type (Fig 2.2(f)): the reason may be there are too much groups or inaccurate measurements. At this time we ought to re-collect and collate the data.

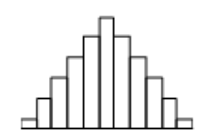

(a)Normal

(d)Lonely island

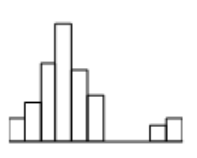

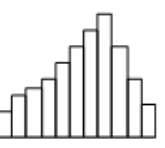

(b)Deviation

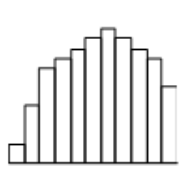

(e)Flat

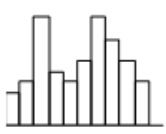

(c)Bimodal

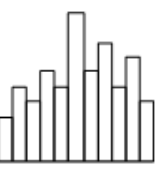

(f) Sawtooth
Fig 2.3. Histogram classification

\subsection{Quality Control Charts}

Quality control charts are used to monitor the production process whether is under control. There are center line (CL), the up control line (UCL) and the lower control line (LCL) on the quality control charts. If the points on the control chart fall within the control lines, indicating that the entire production process is stable and controllable. If the point falls outside the control lines or the arrangement of points between the upper and lower control lines are not random, indicating that the entire production process is abnormal.

\subsubsection{Control Chart Analysis}

(1)There are one or more points beyond the control limits, or although the points are not out of bounds, the arrangement of points is not random. All these features are the main evidences that the system is out of control. Therefore, this situation should be marked in order to investigate the reasons and take corrective measures. National standard of criteria sentenced to abnormal is shown in figure 2.4:

(a): If the points are out of bounds, they are sentenced to abnormal;

(b): Nine consecutive points falls on the same side of the centerline;

(c): Fourteen consecutive points alternate up and down the centerline;

(d): Six consecutive points decrease progressively or increase progressively;

(e): There are two points in three consecutive points fall 
outside the area $\mathrm{B}$ which fall on the same side of the centerline;

(f): There are four points in five consecutive points fall outside the area $\mathrm{C}$ which fall on the same side of the centerline;

(g): Fifteen points fall on the area $\mathrm{C}$ which are up and down the center line;

(h): Eight points are on both sides of the center line. But there are no points falling on the area $\mathrm{C}$.

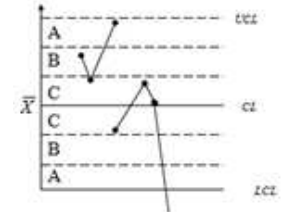

(a)

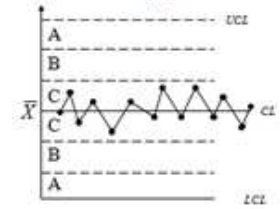

(c)

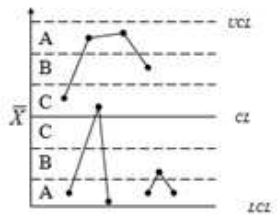

(e)

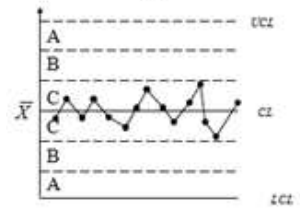

(g)

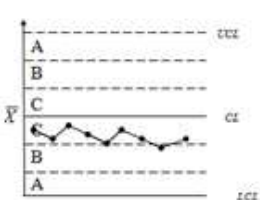

(b)

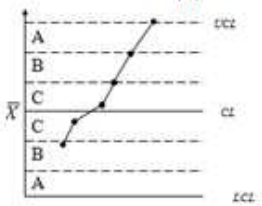

(d)

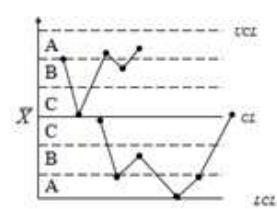

(f)

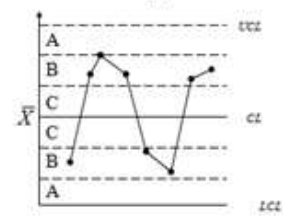

(h)
Fig 2.4. Quality control charts

\subsubsection{The Basic Types of Control Charts}

Classified by characteristics of the product quality, control charts can be divided into the measured value control chart and the numerical value control chart.

Measured value control chart include mean-range control chart ( $\bar{x}-R$ chart), median-range control chart $(\tilde{x}-R$ chart $)$, single value-moving range control chart $\left(x-R_{S}\right.$ chart), mean-standard deviation control chart ( $\bar{x}-S$ chart).

Numerical value control chart include nonconforming rate control chart ( $p$ chart), number of nonconforming control chart ( $p^{n}$ chart), defects per unit control chart ( $u$ chart), defects control chart ( $c$ chart).

\subsubsection{Design Principles of Control Chart}

(1)Normality assumption: The premise of quality control chart is assuming that fluctuations of the quality characteristics value in the production process obey normal distribution.

(2)Criterion of $3 \sigma$ : If the quality feature values $X$ obey normal distribution $N\left(\mu, \sigma^{2}\right)$.According to the nature of the normal distribution:

$$
P\{\mu-3 \sigma<X<\mu+3 \sigma\}=99.73 \%
$$

$(\mu-3 \sigma, \mu+3 \sigma)$ is the actual range of X. Based on this principle, If the design of the control chart for $\mathrm{X}$, centerline $C L=\mu$, upper and lower control limits are respectively $U C L=\mu-3 \sigma, L C L=\mu+3 \sigma$.

(3)Take the $\bar{x}-R$ control chart for example, the control limits of $\bar{x}$ is calculated by the following formula:

$$
\begin{aligned}
U C L_{\bar{x}} & =\bar{x}+A_{2} \bar{R} \\
& = \\
L C L_{\bar{x}} & =\bar{x}+A_{2} \bar{R}
\end{aligned}
$$

$\bar{x}$-The overall average $\bar{R}$-Sample mean poor

$A_{2}$-Coefficient, determined by the capacity of sub-group

The control limits of ' $R$ ' are calculated by the following formula:

$$
\begin{aligned}
& U C L_{R}=D_{4} \bar{R} \\
& L C L_{R}=D_{3} \bar{R}
\end{aligned}
$$

\begin{tabular}{|c|c|c|c|c|c|c|c|c|c|c|c|c|c|c|c|c|}
\hline \multirow{3}{*}{$\begin{array}{l}\text { Sample } \\
\text { sizen }\end{array}$} & \multicolumn{3}{|c|}{ Mean I chart } & \multicolumn{6}{|c|}{ standard deviation chart } & \multicolumn{7}{|c|}{ Range charts } \\
\hline & \multicolumn{2}{|c|}{$\begin{array}{l}\text { Control } \\
\text { coefficient }\end{array}$} & \multirow{2}{*}{$\begin{array}{c}\text { limits } \\
A_{3}\end{array}$} & \multicolumn{2}{|c|}{$\begin{array}{l}\text { Centerline } \\
\text { coefficient }\end{array}$} & \multicolumn{4}{|c|}{ Control limits coefficient } & \multicolumn{3}{|c|}{ Centerline coefficient } & \multicolumn{4}{|c|}{ Control limits coefficient } \\
\hline & $A$ & $A_{2}$ & & $C_{4}$ & $1 / C_{4}$ & $B_{3}$ & $B_{4}$ & $B_{5}$ & $B_{6}$ & $d_{2}$ & $1 / d_{2}$ & $d_{3}$ & $D_{1}$ & $D_{2}$ & $D_{3}$ & $D_{4}$ \\
\hline 2 & 2.121 & 1.880 & 2.659 & 0.7979 & 1.2533 & 0 & 3.267 & 0 & 2.606 & 1.128 & 0.8865 & 0.853 & 0 & 3.686 & 0 & 3.267 \\
\hline 3 & 1.732 & 1.023 & 1.954 & 0.8862 & 1.1284 & 0 & 2.568 & 0 & 2.276 & 1.693 & 0.5907 & 0.888 & 0 & 4.358 & 0 & 2.574 \\
\hline 4 & 1.500 & 0.729 & 1.628 & 0.9213 & 1.0854 & 0 & 2.266 & 0 & 2.088 & 2.059 & 0.4857 & 0.880 & 0 & 4.698 & 0 & 2.282 \\
\hline 5 & 1.342 & 0.577 & 1.427 & 0.9400 & 1.0638 & 0 & 2.089 & 0 & 1.964 & 2.326 & 0.4299 & 0.864 & 0 & 4.918 & 0 & 2.114 \\
\hline 6 & 1.225 & 0.483 & 1.287 & 0.9515 & 1.0510 & 0.030 & 1.970 & 0.029 & 1.874 & 2.534 & 0.3946 & 0.848 & 0 & 5.078 & 0 & 2.004 \\
\hline 7 & 1.134 & 0.419 & 1.182 & 0.9594 & 1.0423 & 0.118 & 1.882 & 0.113 & 1.806 & 2.704 & 0.3698 & 0.833 & 0.204 & 5.204 & 0.076 & 1.924 \\
\hline 8 & 1.061 & 0.337 & 1.099 & 0.9650 & 1.0363 & 0.185 & 1.815 & 0.179 & 1.751 & 2.847 & 0.3512 & 0.820 & 0.388 & 5.306 & 0.136 & 1.864 \\
\hline 9 & 1.000 & 0.337 & 1.032 & 0.9693 & 1.0317 & 0.239 & 1.761 & 0.232 & 1.707 & 2.970 & 0.3367 & 0.808 & 0.547 & 5.393 & 0.184 & 1.816 \\
\hline 10 & 0.949 & 0.308 & 0.975 & 0.9727 & 1.0281 & 0.284 & 1.716 & 0.276 & 1.669 & 3.078 & 0.3249 & 0.797 & 0.687 & 5.469 & 0.223 & 1.777 \\
\hline
\end{tabular}

$D_{3}, D_{4}$ - Coefficient, determined by the capacity of sub-group

$A_{2}, D_{3}, D_{4}-$ can be found from the following table.

Table 2.1. The coefficient table of measured value control chart 


\subsection{Process Capability Index}

Now quality management emphasizes the process capability. Only the stable process can continue to provide qualified products. We can evaluate the overall process with the capability index. There we need to distinguish two kinds of capability index. One is the critical process capability index that describes the potential and process capability. This needs to consider the location of the process. Another is the potential capability index that describes the so-called feature of the critical capability. This only considers the dispersion degree of the process. Position does not affect the results This value gives a comparison between specification tolerances and process discrete width. Process discrete width is defined as the width of $99.73 \%$ of the measurement data that falls in.

\subsubsection{The Calculation of Process Capability Index}

(1)Process capability index of bilateral tolerance situation

When tolerance center coincides with the center of the distribution, calculate the potential capability index $C_{p}$

$$
C_{p}=\frac{\text { Tolerance }}{\text { Process spread }}=\frac{T}{6 S}
$$

When tolerance center does not coincide with the distribution center, process capability index calculated at this time does not reflect the actual situation that has a deviation, then need to calculate the potential capability index $C_{p k}$

$$
C_{P K}=\min \left\{C_{P K U}, C_{P K L}\right\}
$$

(2)Process capability index of unilateral tolerance situation

If emphasis the requirements of the upper specification limit but do not emphasis the lower one, the process capability index is calculated as follows:

$$
C_{P K U}=\frac{U S L-\bar{x}}{3 S} \quad \bar{x}<U S L
$$

$C_{P K U}$-Upper unilateral process capability index, when $\bar{x} \geq U S L, C_{P K U}=0$

If emphasis the requirements of the lower specification limit but do not emphasis the upper one, the process capability index is calculated as follows:

$$
C_{P K L}=\frac{\bar{x}-L S L}{3 S} \quad \bar{x}>U S L
$$

$C_{P K L}$ - Lower unilateral process capability index, when $\bar{x} \leq L S L, C_{P K L}=0$

\subsubsection{Analysis of Process Capability Index}

Analysis of process capability index is shown in the following Tab2.2
Tab 2.2. Analysis of process capability index

\begin{tabular}{ll}
\hline$C p \geq 1.67$ & $\begin{array}{l}\text { The process capability is too high } \\
\text { The process capability is full. This said that } \\
\text { technology management skill has been very good, } \\
\text { should be maintained. } \\
\text { The process capability is poor. This said that } \\
\text { technology management skill has been inadequate, } \\
\text { should be raised. } \\
\text { The process capability is scarce. This said that } \\
1.0>C_{p} \geq 0.67\end{array}$ \\
$\begin{array}{l}\text { technology management skill has been poor, should } \\
\text { be improved immediately. } \\
\text { Process capability is seriously insufficient. This said } \\
\text { that we should take urgent measures, shutdown } \\
\text { overhaul when necessary. }\end{array}$ \\
\hline
\end{tabular}

\subsubsection{Non-Normal Distribution Case}

The above calculation of the process capability index is based on the data were normally distributed. However, in practice there are many known or unknown data distribution in addition to the normal distribution. When we encounter other distribution, we must find the quantile of upper $3 \sigma$ and lower $3 \sigma$, computation formula is as follows:

When bilateral tolerance,

$$
\begin{aligned}
C_{P} & =\frac{U S L-L S L}{X_{99.73 \%}-X_{0.27 \%}} \\
C_{P K} & =\min \left\{\frac{U S L-\mu}{X_{99.73 \%}-\mu} ; \frac{\mu-L S L}{\mu-X_{0.27 \%}}\right\}
\end{aligned}
$$

When unilateral tolerance,

When only require the upper limit of the tolerance,

$$
C_{P K}=\frac{U S L-\mu}{X_{99.73 \%}-\mu}
$$

When only require the lower limit of the tolerance,

$$
C_{P K}=\frac{\mu-L S L}{\mu-X_{0.27 \%}}
$$

$X_{99.73 \%}$ - the upper limit of departing from the scope $X_{0.27 \%}$ - the lower limit of departing from the scope

When it is normal distribution, $\left.\begin{array}{c}X_{99.73 \%} \\ X_{0.27 \%}\end{array}\right\}=\mu \pm 3 \sigma$

When having multiple sampling analysis, we ought to calculate the estimated value $\hat{X}_{99.73 \%}, \hat{X}_{0.27 \%}$ to replace the $X_{99.73 \%,} X_{0.27 \%}$.

In the modern production management process of enterprise, product quality control is very important. Quality is the life of enterprise. Whether using advanced quality control technologies is related to the survival of enterprises. The method of statistical process control is very important in the quality control. But the traditional calculation method of statistical process control is inefficient. The use of the function of Q-das software such as collection, monitoring, management, assessment, reporting, archiving to carry on statistical process control is a fast and efficient method. 


\section{Examples}

Now measure a shaft diameter, size requirement is $14.07_{-0.01}^{0.005} \mathrm{~mm}$.Data are as follows:

Tab 3.1. Measurement data of the shaft diameter

\begin{tabular}{llllll}
\hline 14.0681 & 14.0688 & 14.0675 & 14.0695 & 14.0692 & 14.069 \\
14.0666 & 14.068 & 14.0668 & 14.0661 & 14.0675 & 14.0677 \\
14.0681 & 14.068 & 14.0688 & 14.0703 & 14.0676 & 14.0685 \\
14.0699 & 14.0671 & 14.0692 & 14.0677 & 14.0685 & 14.0671 \\
14.0669 & 14.0657 & 14.0683 & 14.0678 & 14.0657 & 14.0659 \\
14.0679 & 14.0685 & 14.068 & 14.0674 & 14.0681 & 14.0661 \\
14.0677 & 14.0675 & 14.0687 & 14.0693 & 14.0658 & 14.0697 \\
14.0689 & 14.0674 & 14.068 & 14.0667 & 14.0675 & 14.0664 \\
14.0696 & 14.067 & 14.0702 & 14.0661 & 14.0686 & 14.069 \\
14.0687 & 14.07 & 14.0675 & 14.0696 & 14.0679 & 14.0675 \\
14.0668 & 14.0682 & 14.0695 & 14.0692 & 14.0691 & 14.0672 \\
14.0654 & 14.0698 & 14.0656 & 14.0684 & 14.067 & 14.0664 \\
14.0661 & 14.0673 & 14.0695 & 14.0667 & 14.068 & 14.0683 \\
14.0681 & 14.0669 & 14.0671 & 14.0676 & 14.0684 & 14.0697 \\
14.0673 & 14.0686 & 14.0678 & 14.0674 & 14.0687 & 14.0694 \\
14.0666 & 14.0666 & 14.065 & 14.069 & 14.0691 & 14.0676 \\
14.0688 & 14.0709 & 14.0671 & 14.0689 & & \\
\hline
\end{tabular}

(1)First select process analysis module. Fill the relevant requirements of 'create new characteristics, parts mask' and 'characteristics mask' through 'New File' option under the file menu. As shown in figure 3.1.

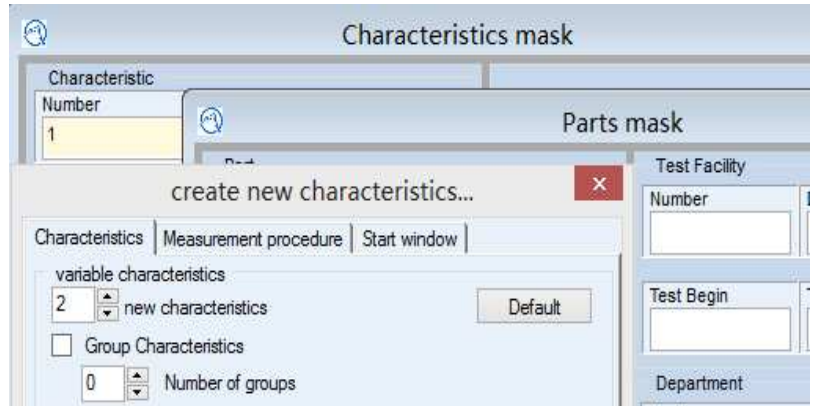

Fig 3.1. Create new characteristics, parts mask'and characteristics mask.

(2)Input data through Values mask import data from an Excel spreadsheet. It can also connect the measurement device to computer through the communication data cable, entry data automatically. As shown in figure 3.2.

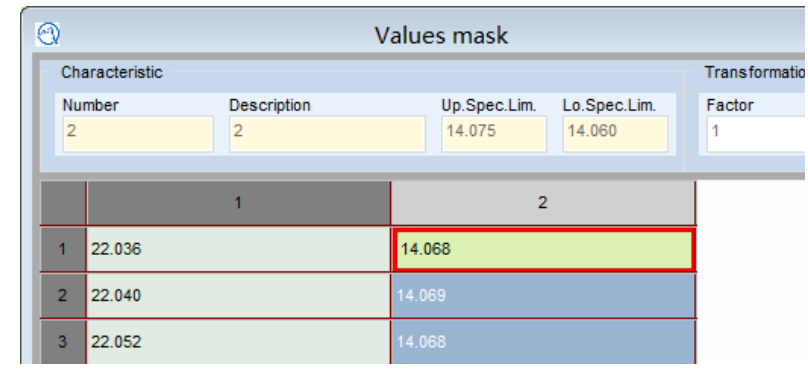

Fig 3.2. Values mask
(3)First, select the evaluation criteria according to different standards. Then the software will draw chart automatically such as value chart individuals, histogram, control charts, etc. Calculate the statistics, process capability index. Make an analysis such as histogram distribution pattern, process capability analysis. Finally Q-das can produce different forms of research report for producer. As shown in figure3.3. As we can see, at this stage, the shaft machining completely meets the requirements. Production and process do not require adjustment. It can continue to produce but still need a real-time detection, just in case.

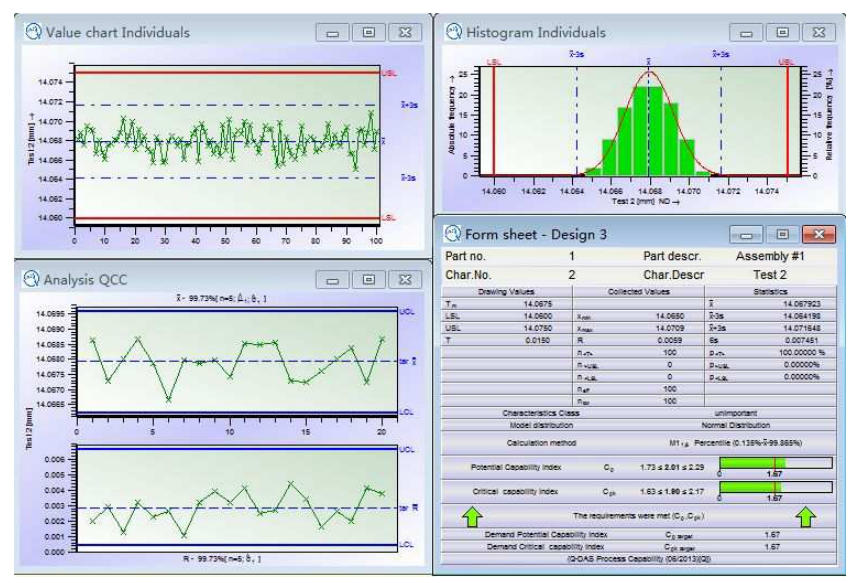

Fig 3.3. Research report

\section{Conclusion}

This article describes some of the commonly used statistical quality control methods and Q-das software applications. Reliable statistics are a key tool for process control assessment. Q-das use efficient and reliable methods to provide a convenient for the quality assessment for industrial manufacturing. Q-das software also has more evaluation criteria settings. Users can set according to different needs. The application of Q-das software for statistical quality control has many advantages. For example, it has unified evaluation criteria of SPC, MSA for major customers. So that it has avoided an inconsistent result that is due to the assessment of different criteria for the manufacturing process. Q-DAS software input data automatically and complete the calculation and analysis of $C_{p}, C_{p k}$. This improve the accuracy of measurement results. The workers will have a more comprehensive view of the state of the production process.

\section{References}

[1] Tian Xuemin, Cao Yuping. Research Status and Prospect of statistical process, control. Journal of China University of petroleum (Natural science edition), 2008, 32(5):175-180.

[2] Jiang Wei. The applications and development trends of Q-DAS software in the, quality control of the production. Automotive Technology, 2009(2). 
[3] Technology and Research Center of China Automotive Company translate. Statistical Process Control (SPC) Reference Manual. Revision Working Group of Chinese version QS-9000 and supporting manual, 2000.5, 2nd Edition.

[4] Long Kejun. Research and Application of the Computer-aided statistical process control (SPC). Technology Vision, 2013(31), 83-84.

[5] Gao Qisheng. Application of Quality Engineering and Control. PhD thesis of Northeastern University, 1999.10.

[6] Zhao Xu. Process monitoring and quality control methods based on statistical, $\mathrm{PhD}$ thesis of Shanghai Jiaotong University, 2006.11.

[7] Zheng Weiwei. Intelligent system of process quality control and method. PhD thesis of Northwestern Polytechnical University, 2006.11.

[8] Li Mengqing, Chen Zhixiang. Comprehensive identification methods of the trend patterns of quality control chart [J].Journal of Huazhong University of science, 2000, 28(5):24-26.

[9] Liu Guangdi. Quality Management [M], Beijing, Tsinghua University Press, 2003.

[10] Qian Xiyuan, Jing Jianfen, Hou Xuxian. Research of statistical process control (SPC) and its application [J]. Computer Engineering, 2004, 30 (19) : 144-145.

[11] Leif Sjoblom. Quality Control by Prevention : A Field Study of an SPC and DOE Implementation[J]. Quality Engineering, 1996-1997, 9(1):51-63. 